\title{
Visualization of the nucleus and nuclear envelope in situ by SEM in tissue culture cells
}

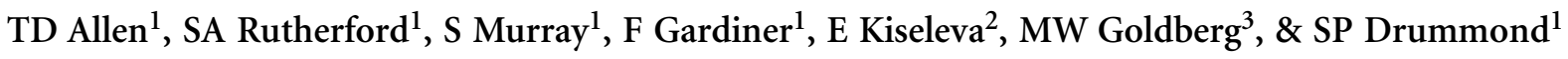

\begin{abstract}
${ }^{1}$ Paterson Institute for Cancer Research, University of Manchester, Wilmslow Road, Withington, Manchester M20 4BX, UK. ${ }^{2}$ Institute of Cytology and Genetics, Russian Academy of Sciences, Novosibirsk, Russia. ${ }^{3}$ School of Biological and Biomedical Sciences, Durham University, South Road, Durham DH1 3LE, UK. Correspondence should be addressed to T.D.A. (tallen@picr.man.ac.uk).
\end{abstract}

Published online 10 May 2007; doi:10.1038/nprot.2007.139

\begin{abstract}
Our previous work characterizing the biogenesis and structural integrity of the nuclear envelope and nuclear pore complexes (NPCs) has been based on amphibian material but has recently progressed into the analysis of tissue-culture cells. This protocol describes methods for the high resolution visualization, by field-emission scanning electron microscopy (FESEM), of the nucleus and associated structures in tissue culture cells. Imaging by fluorescence light microscopy shows general nuclear and NPC information at a resolution of approximately $200 \mathrm{~nm}$, in contrast to the 3-5 nm resolution provided by FESEM or transmission electron microscopy (TEM), which generates detail at the macromolecular level. The protocols described here are applicable to all tissue culture cell lines tested to date (HeLa, A6, DLD, XTC and NIH 3T3). The processed cells can be stored long term under vacuum. The protocol can be completed in $5 \mathrm{~d}$, including $3 \mathrm{~d}$ for cell growth, $1 \mathrm{~d}$ for processing and $1 \mathrm{~d}$ for imaging.
\end{abstract}

\section{INTRODUCTION}

The visualization of vertebrate nuclei by field-emission scanning electron microscopy (FESEM) is necessary and informative in determining the presence and function of nuclear structures and proteins $^{1-3}$. It is worth noting that both Xenopus egg-extracts and oocyte nuclei constitute embryonic systems and that data accumulated by studying these systems may not be directly applicable to somatic cells $s^{4,5}$. Therefore, it has also been important to pioneer techniques for the isolation and visualization of amphibian (and mammalian) tissue culture cell nuclei ${ }^{6}$. The protocol included here was originally developed for Xenopus tissue-culture cell nuclei but has proved equally applicable for the visualization of mammalian cell nuclei. To reveal nuclear structure the cells are subjected to a fracturing technique with an adhesive at room temperature $\left(16-24^{\circ} \mathrm{C}\right)$ in which the fracture planes can pass at various planes through detergent-extracted cells, exposing both nuclear surfaces and internal structure in a way not accessed by frozen fracture. This is because fractures through frozen cells 'seek out' planes of mechanical weakness and are limited to the nuclear membrane alone ${ }^{7}$.

Correlation of the presence or absence of nuclear structures with defined biochemical and molecular perturbations has pro- vided quantitative and qualitative insights into the protein requirements for cellular processes. A current area of research is the investigation of the processes and proteins necessary for the assembly of nuclear pore complexes (NPCs); the gateways for all molecular traffic between the nucleoplasm and cytoplasm ${ }^{8}$. To directly visualize nascent NPCs at high resolution our studies have involved the direct surface imaging of the chromosome surfaces of HeLa and DLD cells at well characterized stages of mitosis. Surface imaging using FESEM permits direct observation of nuclear morphologies that are difficult to reconstruct using conventional thin-section transmission electron microscopy (tsTEM).

This procedure is applicable for the surface imaging of tissue culture cell nuclei and further protocols describing our techniques for the visualization of Xenopus oocyte nuclei and nuclei formed in cell-free extracts of Xenopus have been published ${ }^{4,5}$. Together with immunolabeling of specific proteins, FESEM provides unrivalled information about the density and distribution of proteins and protein complexes at the nuclear surface.

\section{MATERIALS}

REAGENTS

- Adhesive tape (e.g., Time tape; VWR International, cat. no.817-1505)

- Argon cylinder (high purity; Air Liquide)! CAUTION Cylinders should be fitted by suitably trained staff.

- $\mathrm{CO}_{2}$ cylinder (liquid with dip tube less than 5 p.p.m. water; Air Liquide)

I CAUTION Cylinders should be fitted by suitably trained staff.

- Disodium hydrogen phosphate (VWR International, cat. no. 106576)

-Ethanol (VWR International, cat. no. 101077Y) (see REAGENT SETUP)

- FCS (Sigma) (see REAGENT SETUP)

- Glutaraldehyde EM grade (Fluka, cat. no. 49631) ! CAUTION Toxic, observe MSDS advice (see REAGENT SETUP).

- Gly (Sigma-Aldrich, cat. no. G7403) (see REAGENT SETUP)

- Leibovitz cell culture medium with L-GIn (Invitrogen, cat. no. 21083-027)

(see REAGENT SETUP)

- Osmium tetroxide (Agar Scientific, cat. no. R1017) ! CAUTION Toxic,

observe MSDS advice (see REAGENT SETUP).
- Paraformaldehyde P001/1 (TAAB laboratories) ! CAUTION Harmful, observe MSDS advice (see REAGENT SETUP).

- Potassium dihydrogen phosphate (VWR International, cat. no. 104871)

- Potassium hydroxide (VWR International, cat. no. 105021) ! CAUTION

Corrosive, observe MSDS advice (see REAGENT SETUP).

- Triton X-100 (VWR International) (see REAGENT SETUP)

-Xenopus tissue culture cells

- BSA (Sigma-Aldrich) (see REAGENT SETUP)

- Fish skin gelatin (Sigma-Aldrich, cat. no. G7765) (see REAGENT SETUP)

- Fixative (see REAGENT SETUP)

-L-15 medium (see REAGENT SETUP)

- Primary antibody, as appropriate to research (commercially available or custom made)

- Secondary antibody 10-nm gold conjugate EM grade, as appropriate for primary antibody (GE Healthcare)

-Sorensen's phosphate buffer (see REAGENT SETUP) 
-0.05\% (w/v) trypsin/0.53 M EDTA (Invitrogen, cat. no. 25200-056) EQUIPMENT

-35-mm Petri dishes bacteriological (e.g., BD Falcon; Biotrace International)

-35-mm Petri dishes cell culture (e.g., BD Falcon; Biotrace International)

- Coating unit with chromium target capable of depositing 2-3 nm layers of chromium with a grain size of less than $0.4 \mathrm{~nm}$ (e.g., BOC Edwards) (see EQUIPMENT SETUP)

- Critical point dryer (e.g., BAL-TEC, Liechtenstein) ! CAUTION Observe manufacturer's instructions (see EQUIPMENT SETUP).

- Dumont tweezers 3 Dumostar (Agar Scientific) (see EQUIPMENT SETUP)

- High resolution SEM

- Leica basket stem

- Inverted light microscope (VWR International)

- Inverted phase contrast microscope

- Safety cabinet class II (VWR International)

- Silicon chips $5 \times 5 \mathrm{~mm}^{2}$ (Agar Scientific) (see EQUIPMENT SETUP)

- Writing diamond (Agar Scientific)

-90-mm plastic Petri dishes (VWR International)

- Back scattered electron detector fitted to SEM

- Filter paper Whatman grade no. 50 (VWR International)

- Nescofilm (VWR International)

REAGENT SETUP

Fixative $2 \%$ Paraformaldehyde, $0.01 \%$ glutaraldehyde in PBS. Dissolve paraformaldehyde in buffer by heating up to $80^{\circ} \mathrm{C}$ with agitation, allow to cool, then add glutaraldehyde. Prepare fresh on day of use.
Ethanol 30, 50,70 and 95\%, solutions in $\mathrm{dH}_{2} \mathrm{O}$ and $100 \%$. Gly $0.1 \mathrm{M}$ in PBS. Prepare fresh on day of use.

L-15 medium 55\% L-15 medium, 35\% sterile $\mathrm{dH}_{2} \mathrm{O}, 10 \%$ FCS.

Osmium tetroxide $1 \%$ in Sorensen's buffer.

Potassium hydroxide $10 \mathrm{M}$ in $\mathrm{dH}_{2} \mathrm{O}$.

Sorensen's phosphate buffer $(0.15 \mathrm{M}, \mathrm{pH}$ 7.4) Prepare $0.15 \mathrm{M}$ solutions $\mathrm{KH}_{2} \mathrm{PO}_{4}$ and $\mathrm{Na}_{2} \mathrm{HPO}_{4}$ and mix in the ratio of 19:81, respectively, to give a buffer of $\mathrm{pH} 7.4$.

Triton X-100 $0.5 \%$ in PBS.

BSA $1 \%$ in PBS. Prepare fresh on day of use.

Fish skin gelatin $1 \%$ in PBS. Prepare fresh on day of use.

EQUIPMENT SETUP

Coating unit Attach Argon cylinder. More information on

chromium coating of samples for FESEM is available at

http://www.quorumtech.com/Manuals/Current_Technical_Briefs/

TB-SPUTTER.pdf.

Critical point dryer Attach liquid $\mathrm{CO}_{2}$ cylinder. More information

on critical point drying is available at http://www.emitech.co.uk/ cpd-brief.htm.

Dumont tweezers 3 Sterilize by wrapping in foil and heating in a hot oven at $150{ }^{\circ} \mathrm{C}$ for $24 \mathrm{~h}$

Silicon chips Number chips using the writing diamond. Clean by dipping in a beaker of acetone, and dry with tissue. Rinse with $70 \%$ ethanol, place in a glass Petri dish, wrap in foil and sterilize by heating in a hot oven at $150{ }^{\circ} \mathrm{C}$ for $24 \mathrm{~h}$.

\section{PROCEDURE}

\section{Preparation of nuclei for SEM}

1| Defrost frozen stock of Xenopus cells and warm to $20{ }^{\circ} \mathrm{C}$, add to L-15 medium with serum.

$\triangle$ CRITICAL STEP Use sterile technique in a class II safety cabinet throughout Steps 1-7.

2| Re-suspend and pipette into a $75-\mathrm{cm}^{2}$ cell culture flask. Tighten the cap.

3| Grow Xenopus cells at $20 \pm 5{ }^{\circ} \mathrm{C}$. A polystyrene box kept at room temperature will serve as a suitable incubator.

4| Check cells for growth using an inverted phase contrast microscope and change the medium $24 \mathrm{~h}$ after plating and again 2-3 d later (i.e., $2 \times$ per week).

5| Maintain stocks by sub-culturing when the cells become confluent: remove media, rinse briefly in PBS, replace with $2 \mathrm{~mL}$ $0.05 \%(\mathrm{w} / \mathrm{v}$ ) trypsin/0.53 M EDTA for 1-2 min (tap sides and base of flask to dislodge the cells). Re-suspend trypsinized cells 1:10 in L-15 media and grow a fresh flask. Cell growth for Xenopus tissue culture may vary depending on temperature.

6| For FESEM studies, grow cells on silicon chips in cell culture Petri dishes. Cells should be plated on day 1, medium changed on day 2 and the cells used on day 3 when they should be approximately $50 \%$ confluent. They should be grown on the shiny side of the chip. Check density by observing cells growing in the Petri dishes.

7| Rinse cells on chips briefly with L-15 without serum.

8| Fix cells with $2 \%$ paraformaldehyde, $0.01 \%$ glutaraldehyde in PBS for $10 \mathrm{~s}$. Hold each chip individually with forceps during immersion in fixative.

9| Transfer to a Petri dish containing $0.1 \mathrm{M}$ Gly in PBS for 10 min to quench unreacted aldehyde.

$\triangle$ CRITICAL STEP For the following steps use Dumont 3 tweezers to transfer chips manually into a Petri dish containing the appropriate reagent. Keep the cells upward. Do not allow to dry out at any point.

10| Rinse in PBS.

11) Transfer to $0.5 \%$ Triton $X-100$ in PBS for 30 min.

12| Rinse in PBS.

13| If immunolabeling is required, follow the protocol provided in Box 1 before proceeding to Step 14. Otherwise, continue immediately with Step 14.

14| Fix with 3\% glutaraldehyde in PBS for $1 \mathrm{~h}$ at room temperature.

PAUSE POINT Store at $4{ }^{\circ} \mathrm{C}$ overnight up to 1 week.

15| Wash briefly in Sorensen's buffer. 


\section{BOX 1 | IMMUNOLABELING TIMING 3-4 h}

1. Block with $1 \%$ BSA in PBS buffer, 10-20 min.

2. Prepare a wet chamber using a $90-\mathrm{mm}$ Petri dish containing wet filter paper with Nescofilm on top.

3. Dilute the primary antibody at an appropriate concentration in PBS.

4. Place chip on dry filter paper to dry the back.

5. Place chip on parafilm in wet chamber and quickly pipette on $10 \mu \mathrm{l}$ antibody, incubate for $1 \mathrm{~h}$ at room temperature.

6. Wash in PBS buffer $2 \times 10$ min.

7. Block with $1 \%$ fish skin gelatin in PBS for 10 min.

8. Dilute the secondary antibody gold conjugate at a concentration of 1 in 10 in PBS.

9. Place chip on dry filter paper to dry the back.

10. Place chip on parafilm in wet chamber and quickly pipette on $10 \mu \mathrm{l}$ antibody, incubate for $1 \mathrm{~h}$ at room temperature.

11. Wash three times for $10 \mathrm{~min}$ in PBS.

12. Continue from Step 14 of the main protocol.

16| Post fix in $1 \%$ osmium tetroxide in Sorensen's buffer for $10 \mathrm{~min}$.

$\triangle$ CRITICAL STEP Osmium preserves lipid and phospholipid content and also enhances contrast in the specimen.

17| Wash briefly in $\mathrm{dH}_{2} \mathrm{O}$.

18| Dehydrate through ethanol, 5 min each wash: 30, 50, 70, $95(2 \times)$ and $100 \%(3 \times)$

19| Transfer to critical point drying apparatus containing ethanol as intermediate reagent. Use a suitable holding device.

A Leica basket stem assembly with small baskets is suitable for the Baltec CPD (it may be necessary to shorten the stem slightly to fit in the chamber).
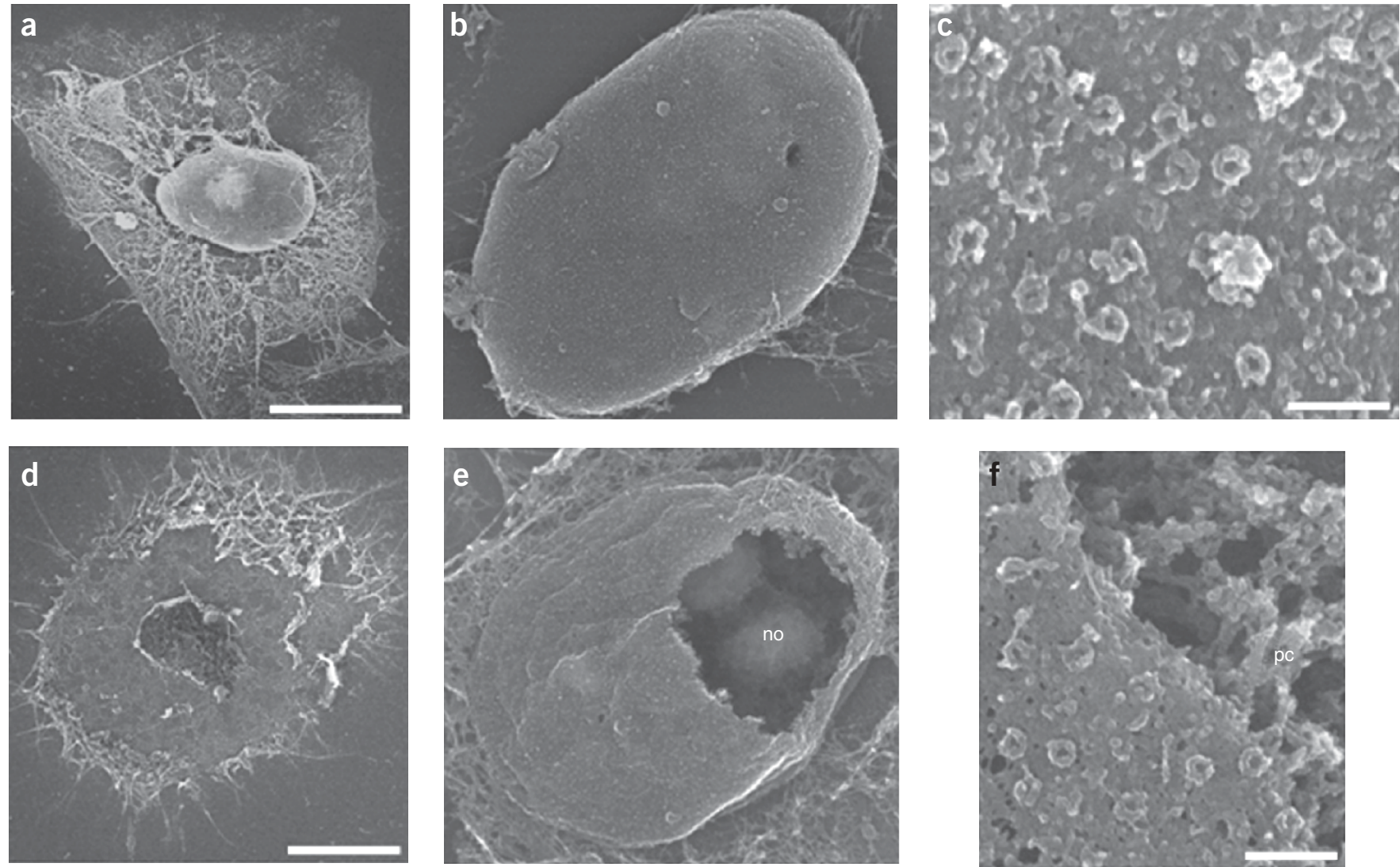

Figure 1 | Visualization of the nuclear surface and interior of tissue culture cells. (a) A nucleus in situ after fracturing and (b) in further detail (whole nucleus) and (c) the cytoplasmic surface of the nucleus showing nuclear pore complexes. (d) Shows a fracture which has traveled through the cell close to the substratum, exposing the nuclear interior, nuclear contents can be seen as differentially condensed chromatin fibers which attach to the inner aspect of the nuclear envelope. Panels (e) and (f) show fractures through the upper aspect of the nucleus, exposing nuclear content such as nucleoli (no) and peripheral chromatin (pc). In (a) and (d) scale bar $=10 \mathrm{~mm}$, in (c) and (f) scale bar $=300 \mathrm{~nm}$. 
Figure 2 | Visualization of immuno-gold labeling at the nuclear surface. After prefixation and extraction, nuclei have been labeled with a monoclonal antibody to a nucleoporin (Nup 107) and secondary labeled with 10-nm colloidal gold. The nuclear surface is exposed by fracture, and coated with $3 \mathrm{~nm}$ of chromium, before examination in the scanning electron microscope (SEM), using secondary and backscatter imaging. (a) Surface topography is apparent from the conventional secondary imaging, and (b) backscatter imaging produces an unequivocal distribution of the gold particles. Both images are acquired simultaneously, and can be superimposed to show the location of the gold (labeling nuclear pore complexes) in situ on the secondary image (inset). Scale bars $=500 \mathrm{~nm}$.

20| Critical point dry from high purity (less than 5 p.p.m. water) liquid $\mathrm{CO}_{2}$.

- PAUSE POINT Store under vacuum for up to 1 week.

21| Dry fracture cells. Attach a $40 \mathrm{~mm}$ length of $13-\mathrm{mm}$ wide adhesive tape to the bench so that it is secured by its ends but the adhesive is uppermost in the middle. Invert chips onto the adhesive and lightly tap with the points of the forceps. Remove chip by carefully pulling from the tape avoiding lateral movements (see Supplementary Video 1 online).

- PAUSE POINT Store under vacuum. Samples should be stable for up to 1 month.

22| Coat samples with 3-nm chromium.

- PAUSE POINT Store under vacuum. Samples should be stable for up to 1 month.

23| Visualize by high resolution SEM.

PAUSE POINT Store samples under vacuum for optimal preservation. ? TROUBLESHOOTING

\section{TIMING}

Steps 1-5: 1 week to grow cells from frozen stocks which can be maintained for up to 6 months

Step 6: $3 \mathrm{~d}$

Step 7-12: $1 \mathrm{~h}$

Step 14: $1 \mathrm{~h}$ or overnight

Steps 15-20: $2 \mathrm{~h}$

Step 21: $10 \mathrm{~min}$

Step 22: $1 \mathrm{~h}$ duration depends on the coating system used and time required to achieve correct vacuum

Step 23: image acquisition: duration depends on number of samples

Immunolabeling (Box 1): 3-4 h

\section{$?$ TROUBLESHOOTING}

Troubleshooting advice can be found in Table 1.

TABLE 1 | Troubleshooting table.

\begin{tabular}{|c|c|c|c|}
\hline Step & Problem & Possible reason & Solution \\
\hline \multirow[t]{5}{*}{23} & Cells insufficiently permeabilized & Cells over confluent & Repeat with less confluent cells on chips (Step 6) \\
\hline & Cells insufficiently permeabilized & Fixation time too long & Be accurate with the $10 \mathrm{~s}$ fixation (Step 8 ) \\
\hline & No cells & $\begin{array}{l}\text { Cells became detached during } \\
\text { processing }\end{array}$ & $\begin{array}{l}\text { Ensure that cells are not too confluent so that they do } \\
\text { not detach in sheets during the protocol (Step 6) }\end{array}$ \\
\hline & No cells & $\begin{array}{l}\text { Entire cells were removed by the } \\
\text { sticky tape rather than fracturing }\end{array}$ & $\begin{array}{l}\text { Use less pressure when attaching the chips to the tape or } \\
\text { use less adhesive tape. Applying tape to only part of the } \\
\text { chip often results in good fractures along the edge } \\
\text { between the fractured and un-fractured areas (Step 21) }\end{array}$ \\
\hline & Very few or no fractures & Tape did not adhere to the cells & Re-fracture and recoat with chromium (Steps 21 and 22) \\
\hline
\end{tabular}
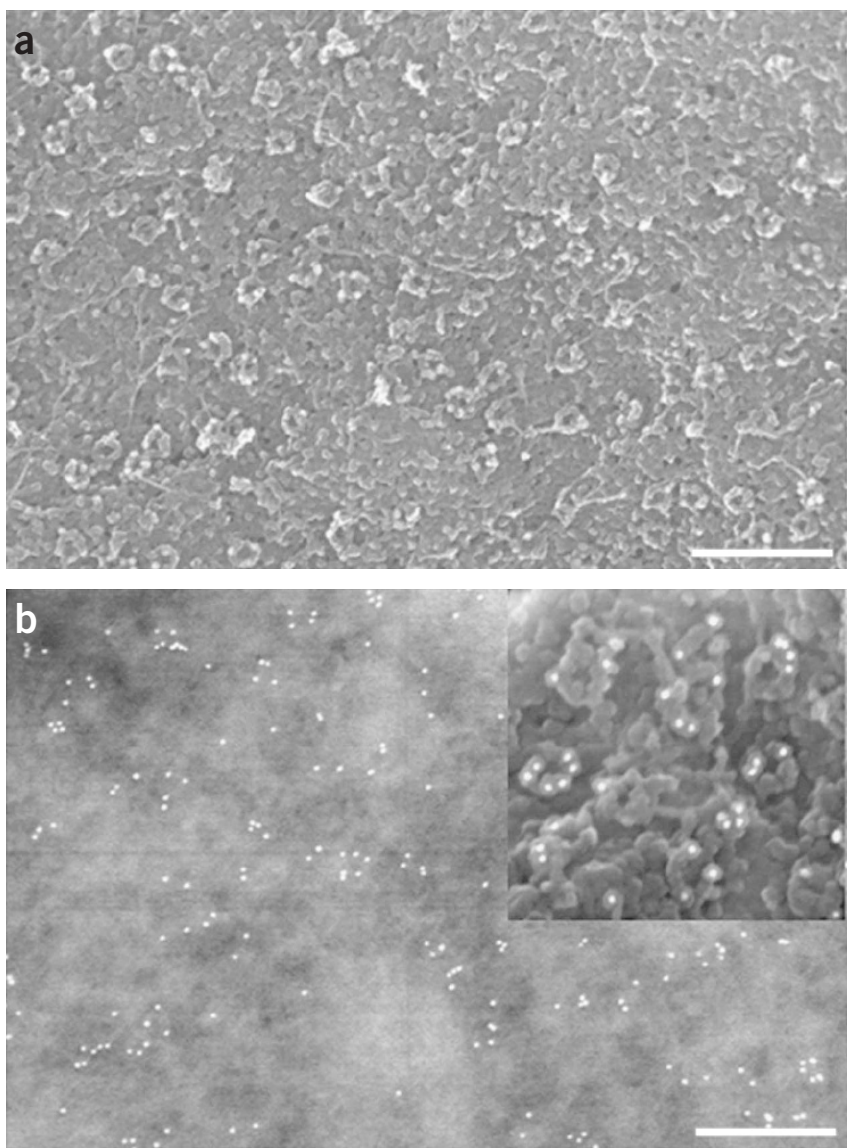
PROTOCOL

\section{ANTICIPATED RESULTS}

This protocol can generate images of the cytoplasmic aspect of the nucleus (Fig. 1a-c) and can reveal internal structures such as nucleoli and chromatin (Fig. 1d-f). The manner in which each cell fractures and consequently the structures that are visible vary between cells. However, cells can be seeded and processed at sufficiently high density to generate statistically significant information. Labeling of cytoplasmically orientated structures of the cell nucleus has been especially important in characterizing the protein components of the NPC (Fig. 2).

COMPETING INTERESTS STATEMENT The authors declare no competing financial interests.

Note: Supplementary information is available via the HTML version of this article.

Published online at http://www.natureprotocols.com

Reprints and permissions information is available online at http://npg.nature.com/ reprintsandpermissions

1. Goldberg, M.W., Wiese, C., Allen, T.D. \& Wilson, K.L. Dimples, pores, star-rings, and thin rings on growing nuclear envelopes: evidence for structural intermediates in nuclear pore complex assembly. J. Cell Sci. 110, 409-420 (1997).

2. Wiese, C., Goldberg, M.W., Allen, T.D. \& Wilson, K.L. Nuclear envelope assembly in Xenopus extracts visualized by scanning EM reveals a transport-dependent 'envelope smoothing' event. J. Cell Sci. 110, 1489-1502 (1997).

3. Drummond, S.P. \& Wilson, K.L. Interference with the cytoplasmic tail of gp210 disrupts 'close apposition' of nuclear membranes and blocks nuclear pore dilation. J. Cell Biol. 158, 53-62 (2002).
4. Allen, T.D. et al. A protocol for isolating Xenopus oocyte nuclear envelope for visualization and characterization by scanning electron microscopy (SEM) or transmission electron micoscopy (TEM). Nat. Protoc. 2, 1166-1172 (2007).

5. Allen, T.D. et al. Generation of cell-free extracts of Xenopus eggs and demembranated sperm chromatin for the assembly and isolation of in vitro-formed nuclei for western blotting and scanning electron microscopy (SEM). Nat. Protoc. 2, 1173-1179 (2007).

6. Drummond, S.P., Rutherford, S.A., Sanderson, H.S. \& Allen, T.D. High resolution analysis of mammalian nuclear structure throughout the cell cycle: implications for nuclear pore complex assembly during interphase and mitosis. Can. J. Physiol. Pharmacol. 84, 423-430 (2006).

7. Kiss, J.Z. \& Staehelin, L.A. Rapid Freezing, Freeze Fracture and Deep Etching (eds. Severs, N.J. \& Shotton, D.M.). (Wiley-Liss, New York, 1995).

8. Peters, R. Introduction to nucleocytoplasmic transport: molecules and mechanisms. Methods Mol. Biol. 322, 235-258 (2006). 